\title{
Synthetic Modelling of Biological Communication: A Theoretical and Operational Framework for the Investigation of Minimal Life and Cognition
}

\author{
Leonardo Bich* \\ IAS-Research Centre for Life, Mind and Society \\ Department of Logic and Philosophy of Science \\ University of the Basque Country (UPV/EHU) \\ Avenida de Tolosa 70, Donostia-San Sebastian, 20018, Spain \\ *Corresponding author: leonardo.bich@ehu.es \\ Ramiro Frick \\ Department of Sociology \\ Universidad Diego Portales \\ Manuel Rodríguez Sur 415, Santiago, Chile \\ and \\ Department of Philosophy \\ Universidad Alberto Hurtado \\ Alameda 1869, Santiago, Chile.
}

\begin{abstract}
This paper analyses conceptual and experimental work in synthetic biology on different types of interactions considered as minimal examples or models of communication. It discusses their pertinence and relevance for the wider understanding of this biological and cognitive phenomenon. It critically analyses their limits and it argues that a conceptual framework is needed. As a possible solution, it provides a theoretical account of communication based on the notion of organisation, and characterised in terms of the functional influence exerted by the sender upon the receiver. It shows that this account can be operationalised in synthetic biology, and that it can supply criteria and guidelines for the design and evaluation of synthetic models.
\end{abstract}

\section{Keywords}

Regulation; synthetic biology; minimal cognition; influence; organisation; biological functions; protocells.

\section{Introduction}

Synthetic biology is a very diversified domain of scientific activity, dedicated to the design, creation, and modification of biological systems and components for human purposes. This field of investigation is characterised by different approaches. They share a common methodological attitude that does not follow rigid protocols but resembles tinkering, and looks for satisficing solutions that work for specific purposes 
$[1,2]$. Much of synthetic biology pursues an approach close to that of engineering, focused on practical applications, and carried out by modifying and using biological material to perform specific tasks [3, 4]. An important part of the community, however, is also involved in developing a deeper understanding of the biological world. It pays special attention to minimal living systems, and to one of the great unanswered questions at the very roots of biology: the origins of life. This practice of knowledge, instead of studying living systems by analysing their parts or by formulating predictive models of their behaviours, aims to understand their functioning by actually constructing (usually a simplified version of) the object of investigation, and by studying the properties and behaviours it exhibits [5-7]. A mix of theoretical and heuristic considerations converge in this branch of synthetic biology to pursue this goal. The result is an operational approach where the contents of hypotheses, definitions, and conceptual frameworks can inspire research and be used, manipulated, and tested in the laboratory [8].

Biological properties are not the only targets of these two branches of synthetic biology. The last decade has been characterised by an increased interest in synthetic models of cognitive phenomena, whose goals vary from the study of minimal cognition and biological communication, to the exploration of the possible contributions of synthetic biology to research in artificial intelligence. The former line of investigation has been focusing on the design of sensory-effector mechanisms and on the study of interacting capabilities in protocells [9-12]. The latter has been pursued by focusing either on information technologies that realise computation through biochemical systems [13], or on embodied artificial intelligence grounded in biochemistry [14].

One of the most promising lines of theoretical and experimental research in this area focuses on the phenomenon of biological communication. Communication is investigated by exploring the possibilities opened by the interactions between artificial and natural cells. The relatively recent recognition that life, even at the unicellular level, does not occur in isolation, but is characterised by collective phenomena, has already been put to work by synthetic biologists in the study of the origins of life [15-17]. Studying communication is particularly relevant in this context in order to understand the collective dimension of the (most basic) biological and cognitive world. For this reason, it has been the object of several conceptual and experimental works in synthetic biology [10-12, 18]. Moreover, synthetic models of communication have even been proposed and employed as evaluation tools for synthetic biology [19, 20].

The aims, scope, methodology, and conceptual foundations of this enterprise are still in course of definition. One of the main problems it faces is the lack of a precise conceptual framework applicable to synthetic biology, capable of capturing the distinctive character of communication and its differences from other types of cognitive interactions. A theoretical and epistemological analysis is needed, and this paper aims to provide it by addressing the conceptual issues underlying research in this area. The purpose is twofold. In the first place, it aims to discuss the scope and relevance of the synthetic models of communication available, besides and beyond their capability to realise successful interactions between protocells and natural cells. In the second place, the goal is to provide a precise theoretical framework to address the phenomenon of communication and to offer suggestions and guidelines to expand or re-orient the existing models, and to design new experiments.

To do so, the paper proceeds as follows. Section 2 discusses the conceptual foundations of the synthetic modelling of cognitive phenomena. It introduces a theoretical framework for minimal cognition applicable at the level of organisation that is characteristic of the phenomena investigated by synthetic 
biology. It also discusses the criteria to evaluate the relevance of interactive models in this field. In Section 3, three synthetic models of communication are analysed. Their virtues and limits in contributing to a deeper understanding of communicative phenomena in biology are discussed. Section 4 focuses on the general notion of biological communication and provides a theoretical account, based on the notion of influence and framed in organisational terms. The goal is to capture the distinctiveness of the phenomenon of communication with respect to other dimensions of minimal cognition, and to supply operational criteria for its investigation in synthetic biology. Finally, in Section 5, the implications of this framework for the evaluation and design of synthetic models are discussed.

\section{Requirements for the Study of Minimally Cognitive Phenomena through Synthetic Models}

Cognition is a wide and diversified biological phenomenon, closely related to, and often identified with, the adaptive interactions between organisms and their environments. However, not all cognitive phenomena have the same pertinence and practical relevance when the goal is to study the relationship between synthetic biology and cognitive science. For example, the debate on cognition in most cases is focused on the study of organisms with nervous systems. However, a characterisation of cognition in terms of properties of the nervous system would not be particularly useful for studying cognitive phenomena at the level of protocells and minimal cells. The choice of the domain of investigation, synthetic biology, strongly constrains the range of cognitive phenomena that can be studied and the types of properties that can be modelled. Moreover, the specificity of this field requires framing the theoretical question of cognition at a level that is relevant for synthetic approaches. Of interest in this context, in fact, is not the whole range of cognitive properties and phenomena, but only those minimal ones that are realised by means of biochemical and biomolecular mechanisms, and can be operationalised through synthetic models (e.g. protocells). Therefore, a theoretical account of minimal cognition as realised in basic living systems would be the most pertinent in this domain.

A theoretical framework that is particularly relevant to the understanding of cognition in this specific scenario is the organisational one, based on the notion of autonomy [21-24]. It has often been applied in synthetic biology to investigate the origins of life and minimal life [25-27]. According to this approach, biological systems are organised in such a way that they are capable of self-producing and selfmaintaining while in constant interaction with the environment. This capability to viably interact with the environment has often been understood as minimally cognitive. Cognition has been characterised in terms of the interactions a living system can enter and the resulting modifications it can undergo without losing its identity [22, 28-31], without necessarily requiring the presence of a nervous system. Within this perspective, a theoretical account has been recently proposed by Bich and Moreno with the precise aim of tackling the issue of the origin and synthetic modelling of minimal cognition [32]. It focuses on the specific features of the minimal biochemical and biomolecular mechanisms underlying the cognitive capabilities of unicellular systems, such as, for example, chemotaxis and communication. The specific theoretical framework introduced in [32] has later been adopted to study minimal cognition not only in synthetic (protocells) and basic (prokaryotic) unicellular living systems, but also in eukaryotic organisms without nervous systems such as slime moulds, which can exhibit very complex behaviours [33].

The starting point of this approach is to focus on one of the essential aspects of cognition, that can be analysed at the minimal level of biological organisation. It consists in the fact that cognitive systems 
should be able to distinguish between some specific features of their interaction with the environment, and to act accordingly, in such a way as to maintain their viability. This capability emerges when distinctions between different kinds of environmental perturbations make a difference for the organism, due to the role played by mechanisms of self-regulation [32].

Regulation, in this view, consists in the capability of living systems to selectively shift between different available regimes of self-maintenance in response to specific variations, due to the action of dedicated subsystems (specifically sensitive to these features) [34]. The crucial point is that in the presence of regulatory mechanisms the response of a system is the result of an evaluation of perturbations operated by the regulatory subsystems themselves (activation plus action). The latter modulate the constitutive dynamics of a system in such a way that the system as a whole becomes able to cope with the environmental perturbations that triggered the regulatory response in the first place: the organism metabolises a new source of food, changes direction of movement, secretes chemicals to neutralise a lethal substance, etc. By doing so, a regulatory subsystem establishes some categories in the environment (the variations that activate the regulatory mechanism). These categories are actually employed by the system to modify its own internal dynamics in a viable way (through the regulatory action). This capability can be considered cognitive in a minimal sense because in the presence of regulatory mechanisms, the environment becomes a source of specific and recognisable interactions for the system, and not only of indistinguishable perturbations (noise).

This theoretical framework is particularly suitable for applications in synthetic biology. It accounts for cognition at the level of minimal living systems, and it does so in terms of biochemical and biomolecular regulatory mechanisms that can be implemented in protocells or semisynthetic cells. Specifically, it makes explicit two operational requirements for minimal cognition: $(a)$ the realisation of biochemical or biomolecular sensory-effector regulatory mechanisms; and $(b)$ the fact that the activity of these mechanisms should contribute to the overall maintenance and viability of the more comprehensive system that harbours and maintains them (e.g., the cell). The adoption of this theoretical approach, therefore, has important implications, as it narrows down the range of the interactive systems that are pertinent for investigating cognition and communication (requirement $a$ ), and that of the possible fullfledged synthetic realisations that can be considered properly cognitive (requirement $b$ ).
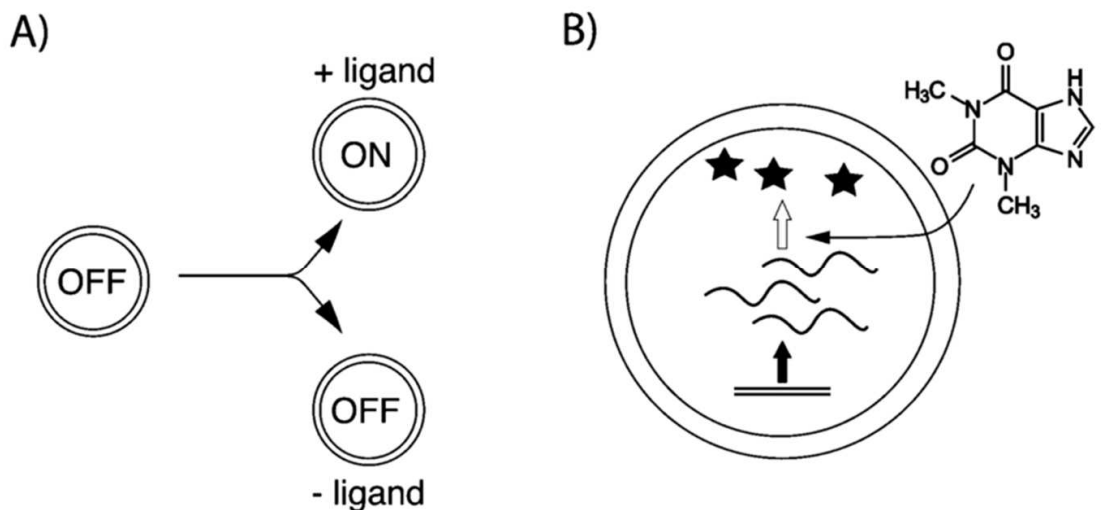
Figure 1. Synthetic model of a sensory-effector biomolecular mechanism. A regulatory mechanism (a) is implemented in a protocell (b) by employing riboswitches that activate RNA transcription from DNA in the presence of a specific ligand [9 reproduced by permission of The Royal Society of Chemistry].

At the same time, this approach makes explicit the type of abstractions that are necessary to realise more simplified synthetic models: that is, narrowing down the scope of the model in order to focus on specific minimal sensory-effector mechanisms, when the model system is a protocell that is not capable of selfmaintaining. An example of this latter approach is constituted by Martini and Mansy's [9] protocells enclosing riboswitches (Figure 1). This experimental model shows how these protocells can sense specific molecules coming from the environment, and respond to them by triggering gene expression and RNA transcription. In this case, the possibilities opened by specific cognitive-like minimal mechanisms can be explored without incurring the current overwhelming difficulties of realising from the bottom up fully fledged autonomous systems harbouring self-maintaining metabolisms.

Satisfying requirements $a$ and $b$ imposes strong constraints on the possible domains of realisation of minimally cognitive or cognitive-like systems. It narrows down the range of systems that are pertinent for realising and investigating cognition and communication to those with a biochemical basis. In doing so, this approach gives wetware-based models a more central role compared to hardware- and softwarebased ones (see [32] for a more detailed discussion of this point). However, it does not commit to one specific biochemical basis, as far as the model systems realise self-maintenance in far from equilibrium conditions.

As will be shown, the requirement of a biochemical basis - or of a domain of realisation that shares some of the properties of the biochemical one - holds not only for synthetic models of minimal cognition, but also for communication. The biological phenomenon of communication relies on cognitive capabilities. As will be shown in the next sections, communicative interactions too, as a subset of cognitive interactions, need to be characterised by taking into account how they are produced by biological systems (requirement $a$ ), and how they contribute to, or affect, the regime of self-maintenance of the biological systems involved in the interaction (requirement $b$ ).

\section{Synthetic Models of Communication}

Among the cognitive phenomena investigated by synthetic biology, biological communication stands out as the object of a thriving line of research, focused on the interactions between artificial (protocells) and natural cells. The main goals of this enterprise are technological and theoretical. The former goals are pursued through the study and implementation of mechanisms of targeted administration of molecules to living cells by means of protocells, and through the development of biochemical information technologies. This line of research is mainly aimed toward medical and industrial applications. The latter goals are related to the phenomenon of biological communication per se, and they are pursued by designing and realising synthetic models of biological intercellular interactions. This second line of research aims to provide insights into the nature and origin of minimal forms of biological communication and cognition by means of artificial systems.

This second type of synthetic approach to communication is being pursued through a hybrid methodology, focused on the interaction between fully fledged minimally cognitive systems (natural cells) and artificial cognitive-like systems, such as, for example, the protocells with riboswitches described in Section 2. It is important to point out that when communication is the target of analysis, the 
main focus of investigation is partly different from the case of individual cognition. The main goal is not only the design and study of viable relationships between a system and its medium (a source of perturbations) but, more importantly, the realisation of successful interactions between cognitive (and/or cognitive-like) systems.

This approach to communication faces specific modelling challenges. In particular, it needs to conform to two types of criteria of relevance for synthetic models, as defined by Damiano and collaborators: phenomenological and organisational [6]. A synthetic model is phenomenologically relevant if it produces, according to explicit parameters, the same phenomenology as a living or cognitive system, regardless of the underlying mechanism, which can vary. In the case of cognition, a model is relevant at the phenomenological level if it produces the same behaviour as a cognitive system, or if it engages in similar interactive dynamics. A synthetic model is organisationally relevant if, according to a specific theory of life and/or cognition, it realises the same organisation as the living or cognitive system that is the object of investigation. In this case, the primary target is not the features of some phenomena or behaviours, but how they are generated.

A synthetic approach to communication, considered as a dimension of cognition, needs to satisfy specific requirements. One of them consists in taking into account the self-regulatory mechanisms at the basis of minimal cognitive capabilities as exhibited by basic living systems (organisational relevance). Yet regulatory mechanisms alone are not enough, insofar as the target is not just adaptivity in a changing environment, but how minimally cognitive systems - that is, autonomous systems endowed with regulatory capabilities - are capable of adaptively interacting/communicating among themselves. Therefore, an external (inter-system) point of view is also required in order to take specifically into account the features of the interactions that minimally cognitive systems undergo among themselves without losing their viability, and how the consequent internal modifications affect the interactive dynamics themselves (phenomenological relevance). Synthetic models should capture this double dimension of communication, and therefore satisfy both organisational and phenomenological criteria of relevance.

Let us consider three examples of synthetic approaches to communication. All employ some mechanism of signal exchanges and consequent activation of effector responses. The first example, from Suda et al. [18], focuses on communication by putting specific attention on the possibilities of applications at the molecular level. The starting idea is that molecular communication can be characterised as a five-step process of encoding, sending, propagating, receiving and decoding information. This process is not necessarily instantiated by cells but also by free artificial molecular devices. According to this view, molecules are energetically very efficient information carriers that, like in the case of ions, can activate membrane channels (intercellular communication), or be carried by molecular motors (intracellular communication), such as in the case of acetylcholine transport along the axon of a neuron. The process of communication starts when the sender produces and stores molecules (encoding), and then releases them (sending), for instance, when a certain concentration threshold is reached. These molecules are then propagated through the environment (passively in the medium, or actively by means of transport motors) from the sender to the receiver. Then, the receiving and decoding steps take place when the carrier molecules bind to the receiver (through a membrane receptor or a free molecule with structural compatibility with the carrier), and the receiver reacts by changing its behaviour or properties (e.g., by undergoing allosteric conformational changes). 
This account of communication focuses primarily on molecular interactions per se, with the aim of developing nanomachines. It does not necessarily take into account how a more comprehensive biological or artificial system realises, harbours, maintains, and employs such molecular mechanisms to engage in communicative interactions. The reason lies in the fact that the goal of this approach is to develop chemical information technologies. The context in which the modelled interactions are characterised as communicative is that of human applications, rather than of interactive biological entities. In this scenario, it is the human user who employs these molecular mechanisms to realise biologically inspired and more efficient processes of human communication.

This approach exhibits two main limits if employed beyond technological applications; that is, to develop, instead, insights into the phenomenon of biological communication per se. In the first place, it does not provide criteria on the basis of which to distinguish communication from other types of specific molecular interactions such as ligand binding. In the second place, it relies on the notion of transfer of information from a sender to a receiver. This aspect is already problematic in itself, due to the set of heavy assumptions that the use of the concept of information carries. Yet it raises even more issues with regards to the possibility of implementing an information-based concept of communication applicable in synthetic biology. One issue that is particularly serious in this regard is how a signal should transmit semantic information. Attributing semantic representational capabilities to protocells and bacteria would introduce more problems than solutions, as it would ultimately require conceiving and designing biochemical mechanisms for the construction of internal semantic representations in these minimal systems. The model in question, however, does not address these issues, and the appeal to information actually remains mostly metaphorical, insofar as a precise characterisation in informational terms has not been provided for each step. The model can be explained instead in terms of molecular interactions alone, such as ligand binding, membrane receptor activation, and allosterically induced conformational change. The use of notions such as encoding and decoding does not seem to provide a deeper understanding of the processes investigated.

A different type of synthetic approach to communication is provided by Rampioni et al.'s simplified model of a synthetic cell sending signals to a natural cell [11]. The model addresses communication in its full dimension as a biological and cognitive process. The underlying idea is to design protocells capable of sending signals and triggering responses in living cells (e.g., a bacterium). A mathematical model is proposed as a first step in this direction. It evaluates the feasibility of building a biochemical synthetic model (Figure 2(a)), by inserting in liposomes biomacromolecules capable of realising the necessary steps for the production of a signal molecule (e.g., $\mathrm{N}$-acyl-homoserine lactones). This molecule, once sensed by the receptor of a natural cell, would in turn trigger processes of protein synthesis in the latter. In doing so, this approach explores the possibilities of a one-way interaction. More specifically, it is aimed at the design of synthetic effector mechanisms capable of producing and releasing signals that trigger an actual change in the receiver cell. 
A)

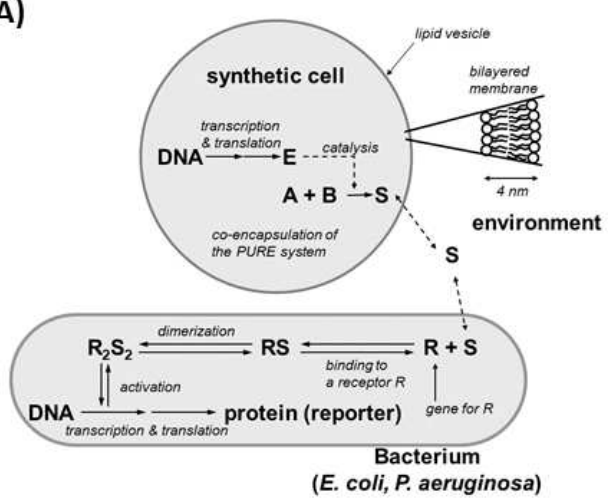

B)

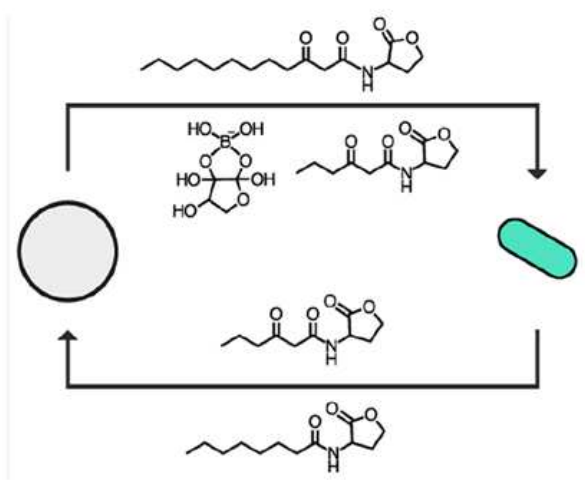

Figure 5. (a) Simplified model of an artificial cell sending signals to a natural cell. The protocell synthetises a signal molecule $\mathrm{S}$ that can be sensed by a natural cell, and trigger protein synthesis (Redrawn, with minor modifications, from [11]. Details of the mechanims can be found in the original publication.). (b) Scheme of a synthetic approach to two-way communication between artificial and natural cells by means of quorum molecules (from [12], https://pubs.acs.org/doi/10.1021/acscentsci.6b00330, further permissions related to the figure should be directed to the ACS).

While this second example specifically focuses on the feasibility of synthetic communication proceeding from synthetic to natural cells, a more recent model designed by Lentini et al. [12] attempts to realise (the conditions for) a two-way communication (Figure 2(b)). In this case, protocells are endowed with the capability to sense quorum molecules produced by bacteria. These molecules can cross the protocells' compartment and activate transcriptional regulatory binding sites within the protocells. As a result, the activated protocells can produce other signal molecules, which are sensed by bacteria and can even interfere with quorum sensing mechanisms within bacterial communities. These protocells, therefore, can be considered as capable of establishing successful interactions with bacterial cells.

Several comments should be made on these last two models, and more generally on the enterprise of using synthetic biology to investigate biological communication. The models satisfy the criteria of phenomenological and organisational relevance, and they do so by exhibiting successful interactive capabilities by means of biochemical regulatory mechanisms. The model proposed by Rampioni et al. focuses primarily on the effector dimension, while the other realises a whole sensory-effector mechanism. These interactions can be considered cognitive-like if interpreted through the lens of the theoretical framework of minimal cognition proposed in Section 2, as they realise sensory-effector mechanisms, but not self-maintenance.

The fact that these models instantiate cognitive-like interactions, however, does not necessarily mean that they are realising that specific type of interactions that can be characterised as communication. This is where their main limit lies. In fact, not all cognitive interactions are instances of communication, but the models of protocells discussed in this section seem to fail to account for this crucial distinction. The theoretical framework adopted by Rampioni et al. [11], for example, is based on Maturana and Varela's autopoietic theory, and more specifically on an account of communication understood as a "structural coupling" between autonomous systems [35]. On this basis, Rampioni et al. propose to study communication in synthetic biology by focusing on covariances between interacting entities: 
"coordinated" or "coupled" behaviours resulting from dynamical processes of reciprocal perturbations and compensations, in which each system influences the behaviour of the other(s).

However, this account seems too broad. Let us consider, for example, a predator-prey interaction. This well-known phenomenon follows an abstract pattern of mutual influences and compensations similar to the ones instantiated in the two examples of protocells discussed in this section: (1) a crouching lion looking at a gazelle starts running toward it, and in doing so produces an incidental sound; (2) the gazelle hears the sound, looks around, sees the lion, and starts running; (3) the lion starts chasing it; (4) the gazelle adjusts its course in response; (5) the lion in turn adjusts its course to the new path of the gazelle, and so on and so forth. The interacting dynamics of the two systems are coupled. They realise behavioural coordination, which involves cognitive capabilities and results from an interlocked triggering of changes of state between the systems involved. A similar pattern can occur at the intercellular level in phenomena that are closer to those modelled by synthetic approaches, for example between an amoeba predator and a bacterium.

Clearly, these are not instances of communication. Yet the approaches adopted in the synthetic models of protocell-cell interactions discussed earlier in this section would not exclude these cases of noncommunicative coordinated behaviours. By lacking criteria to discriminate between different types of interactions, they seem to fail to address the specificity of the very phenomenon they should contribute to understanding, thus undermining their intended explanatory goals. In their current form, therefore, they might not be up to the task of specifically modelling biological communicative interactions.

\section{An Operational Approach to Communication: The Organisational-Influence Account}

Given the issues exhibited by the accounts discussed in the previous section, a new conceptual framework may be necessary to support the synthetic investigation of communication. A necessary step in this direction is to discuss what biological communication is and what types of phenomena and interactions it includes. The aim is to provide an operational account [8] capable of grounding and orienting theoretical and experimental research on communication in synthetic biology, and of offering guidelines for the evaluation of results. This account should be able to provide conceptual tools to discriminate between communicative and other cognitive interactions (demarcation requirement), and it should be applicable to those mechanisms and phenomena investigated by means of synthetic models (operationability requirement).

It has been shown that communication does not include all types of interactions between minimally cognitive systems, and that it cannot be understood as merely "coordinated" or "coupled" behaviour. Predator-prey interactions, for example, are cases of coordinated behaviour but not of communication. Communication as information transfer is also a problematic criterion and difficult to apply in the context of synthetic biology. So how is it possible to characterise biological communication in such a way as to satisfy both demarcation and operationability requirements, and provide useful guidelines for the design and evaluation of synthetic models?

There is another popular approach to communication that, although it requires some reframing, seems more suitable to be applied to the level of organisation that is relevant for synthetic biology and minimal life. It characterises communication as influence, and more specifically, functional influence [36, 37]. 
The idea is that communication takes place when a signal emitted by a sender triggers a change in the behaviour of the receiver that is functional for the sender itself. In this perspective, in order for a system A to communicate with another system B by means of a signal S, it is necessary but not sufficient that $\mathrm{S}$ triggers a change of state in $\mathrm{B}$. Additionally, it is necessary that emitting $\mathrm{S}$ has the biological function of triggering such changes of states in B. The functional dimension is essential. It is what allows us to distinguish proper signals from mere cues, and communication from other kinds of interactive phenomena such as a predator-prey system. The noise (S) made by the lion (A), in fact, triggers the escape of the gazelle (B). But it cannot be said to have the function of making the gazelle run away.

The notion of communication as influence was introduced by Dawkins and Krebs [36] in an evolutionary framework according to which what is functional for the sender is interpreted in terms of adaptations: the signal is a functional trait because it allowed the ancestors of the sender to survive and to reproduce at a higher rate than other individuals lacking this trait. Indeed, biological functions have usually been understood in evolutionary terms, as naturally selected effects. However, if they are characterised in these terms, it follows from this approach that any complex interaction between organisms, "no matter how ritualized or similar to known cases of communication," could not be considered to be an instance of communication unless its evolutionary history has been advanced [38]. Accordingly, when promoting the influence approach within the field of bacterial communication, Diggle and colleagues state: "When we see cell A produce a substance $\mathrm{X}$ that elicits a response in cell $\mathrm{B}$, it is tempting to conclude that the substance produced is a signal. [...] to demonstrate that substance $\mathrm{X}$ is a signal and not a cue, it is necessary to show that it evolved owing to the response it elicits." [39, p. 1242].

Framed in this way, this notion has several limits. The question of what communication is and how it takes place in the currently observed systems is conceptually different from the question about how the communicative interaction originated in the first place. Yet this approach seems to conflate these two dimensions. Moreover, in current scientific practice, what most biologists are usually interested in is the current phenomenon of communication, rather than its evolutionary history, which is usually investigated only after the trait in question has been already described as a signal and the interaction that it mediates as an instance of communication. Most importantly, what is particularly problematic for the purposes of synthetic approaches is that the characterisation of communication as a product of natural selection rules out $a$ priori the very possibility of an artificial, non-evolved communication system, making this approach useless in the context of synthetic biology.

Despite all of these problems, however, the influence approach can be reframed and put to work as the conceptual and operational account that is currently missing in synthetic biology, and that is utterly required in order to support synthetic approaches to communication. In its most general form, the influence approach to communication states that a signal is some trait of the sender: $(i)$ whose presence triggers some response in the receiver; and (ii) that has the function of triggering such a response. Although proponents of the influence approach have taken for granted an evolutionary understanding of biological functionality in terms of adaptation and natural selection (the influence approach is sometimes called "the adaptationist approach" [40]), this is not the only possibility. In fact, the influence approach can be reframed in terms of the current organisation of the system rather than in terms of evolutionary adaptations. This operation can be done by grounding the notion of functional influence into the organisational account of biological functions [41] (see also [42]), according to which a function is 
understood as a contribution of a trait to the maintenance of an organisation (e.g., a living cell) that, in turn, contributes to producing and maintaining the trait itself.

By adopting this account, the influence account of communication can be reformulated in organisational terms, in which to say that a signal is functional specifically means that it contributes to the maintenance of the current organisation of the sender, without necessarily appealing to its evolutionary history. In this view, communication in the most basic sense implies that: $(i)$ a receiver responds to a signal emitted by the sender; and (ii) that a signal is a sender's trait that by triggering some response in a receiver, contributes to maintaining the organization that, in turn, is responsible for producing and maintaining the signal trait itself. Operationally speaking, a process of communication is realised when the regulatory mechanisms of a system $\mathrm{A}$ are activated by specific features of their interaction with the environment and they modulate the internal dynamics of $\mathrm{A}$. The regulated system A produces a signal $\mathrm{S}$ that triggers a regulatory action in a second system $B$, the receiver, that changes its own behaviour. The new behaviour of $\mathrm{B}$, in turn, is functional for the sender in the sense that it contributes to the maintenance of $\mathrm{A}$ in the context that activated the regulatory action in the first place. In this scenario, the interaction between $\mathrm{A}$ and B can be said to be both cognitive, as it employs the sensory-effector regulatory mechanisms of each of the systems, and communicative, as it is functional for the sender A. In other words, communication relies on the cognitive (or cognitive-like) capabilities of both systems $\mathrm{A}$ and $\mathrm{B}$. Yet, as an interactive cognitive phenomenon, it is realised in the more encompassing A-B dynamics.

The organisational-influence account of communication has conceptual, theoretical and empirical virtues over the most salient alternatives. It is particularly suitable for applications in an experimental field such as synthetic biology, as it satisfies both operationability and demarcation requirements. Operationally speaking, being based on the notion of influence, this approach is free of the problems related to the application of the concept of information in this domain. It is also more parsimonious, insofar as it does not impose strong requirements for synthetic realisations, such as the design of specific mechanisms for the encoding, transmission, and decoding of information in protocells and artificial cells. It requires only the presence of regulatory sensors and effectors (organisational criterion of relevance), plus the fact that the systems engaged in communication should realise a certain pattern of interaction according to which the response to a signal is functional for the sender (phenomenological criterion of relevance).

Moreover, this framework focuses on the features and behaviours of current systems, regardless of the remote historical evolutionary facts, and thus, it does not exclude a priori the possibility of non-evolved, artificially designed communication systems. For these reasons, this proposal can provide conceptual and heuristic guidelines for, and shed some light on, the present and future experimental work in synthetic biology, where the object of study is artificial systems, such as protocells, which are not the product of evolution.

This theoretical framework does not consider the fact of being the result of evolution as a necessary condition for biological communication. Yet it does not exclude from the study of communication possible synthetic systems that would emerge as the result of artificial evolutionary techniques. It is not in contradiction with evolutionary accounts and it can also provide a theoretical grounding for functions and communication that can account also for those cases in which the interactions are the result of natural selection. As a matter of fact, it has been argued that the evolutionary account actually presupposes the existence of individual organisms that were able to survive and reproduce in their environment, and that 
evolutionary adaptations depend on individual adaptive organisation [41-44]. The organisational framework specifically accounts for the capability of current systems to survive and reproduce in their environments. In this perspective, traits that are selected in the process of evolution are a subset of all the traits to which functions can be attributed in organisational terms. Having a framework that can ground communication without necessarily appealing to evolutionary history is important when the target of the investigation is the characterisation of the phenomenon of communication as it occurs (in the present). The cases of communication analysed in Section 3 are specifically focused on the study of current communicative interactions, and on designing systems that are capable of communicating. Their goal does not consist in producing these capabilities anew, each time, through artificial evolutionary processes subject to natural selection. However, although possible, the latter case would be very demanding in terms of time and resources and would actually respond to a different question: the evolutionary history of a communicative trait or system, rather than how communication works.

At the same time, this proposal exhibits demarcating power, as it can distinguish signals from cues, so that it characterises as instances of communication only a proper subset of all coordinated behavioural interactions, excluding phenomena such as predator-prey interactions. According to this account, the fact that an artificial cell A produces a substance $\mathrm{S}$ that elicits a response $\mathrm{R}$ in a natural cell $\mathrm{B}$ is a necessary but not a sufficient condition for communication in the biological sense. Communication (and saying that substance $S$ is a proper signal and not a cue) requires that $S$ has the biological function of triggering the response $\mathrm{R}$ in $\mathrm{B}$. More specifically, this implies that: $(i) \mathrm{A}$ is a self-maintaining system and selfproducing autonomous systems; and (ii) $\mathrm{R}$ (the receiver's response triggered by $\mathrm{S}$ ) is functional for A; that is, it contributes to the maintenance of $\mathrm{A}$.

\section{Discussion: A Change of Perspective}

This paper addressed the issue of how to investigate minimal cognitive properties and phenomena in synthetic biology. It analysed its requirements, and the theoretical and epistemological tools available. One of the most promising lines of research in this scenario, it has been shown, is constituted by the synthetic modelling of biological communication, which explores the possibilities offered by protocells interacting with living cells.

By analysing the current theoretical scenario and three examples of synthetic models of communication, it has been shown that a precise account of communication able to capture the specificity of this phenomenon, and that is capable of distinguishing it from other types of cognitive interactions, is needed. Considering that explicit or implicit conceptual frameworks play an important role in the identification and design of the interactive dynamics to be investigated, the lack of a precise framework may have negative consequences, seriously affecting the scope and explanatory power of synthetic models.

To solve this issue, a theoretical account of communication as influence has been proposed: the organisational-influence account. It can be operationalised into synthetic models by realising systems endowed with regulatory mechanisms and capable of functionally influencing one another. The adoption of this framework has several implications for the modelling of communicative interactions, ultimately entailing a change of perspective.

In the first place it enhances the specificity of models. It provides criteria that allow narrowing down the object of investigation to that subset of cognitive or cognitive-like interactions that are relevant for the 
study of the phenomenon of biological communication, and putting them into focus. This is something that other theoretical frameworks seem to fail to achieve. According to this perspective, to study communication it is necessary to look for how the sender of a signal influences the behaviour of the receiver in such a way that the receiver, in turn, contributes to the viability of the sender. The focus, therefore, is on those interactions between senders and receivers that are functional for the senders.

In the second place, this conceptual shift requires complexifying the models available, through the establishment of a specific type of functional loop of interactions that proceeds from the sender to the receiver and back to the sender. This type of loop differs from the interaction schemes realised in the models developed by Rampioni et al. [11] and Lentini et al. [12], and analysed in Section 3. These latter models rely mainly on linear interactions, in which a system affects another: from senders (protocells) to receivers (cells) in the first one, and with the additional capability of senders (protocells) to sense the medium in the second one (an environment-sender-receiver interaction). The main objective of these models is to realise successful interactions that trigger a response in the receiver. Yet, in their current form, they do not take into consideration the functional relationship that is characteristic of communication as a biological phenomenon, and they cannot differentiate it from other even very complex interactions, such as those occurring in the dynamics of a predator chasing prey.

In the third place, from the operational point of view, to realise synthetic models of communication understood as an influence exerted between artificial and natural cells, it is possible to proceed in two ways: with either cells or protocells as senders, respectively. In the first case, the focus is on how a cell can functionally influence a protocell. Models would need to employ protocells that can respond to signals emitted by cells. However, rather than just trigger a reaction in the receiver, the signal should change the behaviour of the protocell in such a way as to contribute to the maintenance of the sender cells. This functional response by the protocell may be achieved, for example, by releasing a useful protein that the cell is missing, or a metabolite not available in the environment.

The second approach takes protocells as senders. It allows exploring specific aspects of communication, or communication-like phenomena, by investigating how protocells can influence the behaviour of natural cells in a way that can be considered somehow functional for the protocell, despite the fact that the latter is not capable of achieving full-fledged metabolic self-maintenance. A possibility can be to modify the behaviour of the receiver cells in such a way as to contribute to making the protocell more stable in a given environment (e.g., by providing different types of membrane components), or to enable new functions (e.g., by providing new enzymes and metabolites that can be employed by the protocell to perform new operations).

However, the possibilities opened by synthetic models are not restricted to interactions between natural cells and protocells. For example, an additional line of investigation, of special interest for the origins of life, could be to explore the range and possibilities offered by the communication-like loops between protocells in a prebiotic environment, without involving living cells.

\section{Concluding Remarks}

The adoption of a theoretical account, such as the organisational-influence one, that can be operationalised by employing and redesigning already available protocells with sensory-effector capabilities, can provide a conceptual and heuristic guideline for the investigation of biological 
communication and of its minimal instances. By making explicit the distinctive features of communicative phenomena, it provides tools and criteria to reframe the interactive dynamics between natural and artificial cells in order to model behaviours that are relevant for understanding the nature and roots of biological communication. In particular, this approach puts into evidence the necessity for modellers to shift their attention from designing protocells that can interact with cells, to protocells that can participate in functional loops with cells or among themselves.

\section{Acknowledgements}

The authors thank Pasquale Stano for redrawing Figure 2(a). This work was funded by the Ministerio de Economia, Industria y Competitividad (MINECO), Spain ('Ramon y Cajal' program, grant RYC-201619798; research project FFI2014-52173-P for LB), by the Basque Government (research project IT 59013 for LB), and by CONICYT, Chile (Doctoral Scholarship 21151293-CONICYT and Supporting Doctoral Thesis Scholarship for RF).

\section{References}

[1] S. Green, "Philosophy of Systems and Synthetic Biology," in The Stanford Encyclopedia of Philosophy, (E. N. Zalta, ed.), https://plato.stanford.edu/archives/win2017/entries/systems-synthetic-biology, 2017.

[2] M. A. O'Malley, "Making Knowledge in Synthetic Biology: Design Meets Kludge," Biological Theory, 4(4), 2009 pp. 378-389.

[3] P. A. Silver and J. C. Way, "Rationalizing Nature," Nature, 509, 2014 p. 166.

[4] F. H. Arnold and J. T. Meyerowitz, "Evolving with Purpose," Nature, 509, 2014 pp. 166-167.

[5] R. Pfeifer and C. Scheier, Understanding Intelligence, Cambridge, MA: MIT Press, 1999.

[6] L. Damiano, A. Hiolle and L. Cañamero, "Grounding Synthetic Knowledge: An Epistemological Framework and Criteria of Relevance for the Scientific Exploration of Life, Affect and Social Cognition," in Advances in Artificial Life, ECAL 2011 (T. Lenaerts, M. Giacobini, H. Bersini, P. Bourgine, M. Dorigo and R. Doursat, eds.), Cambridge, MA: MIT Press, 2011 pp. 200-207.

[7] K. Ruiz-Mirazo and A. Moreno, "Synthetic Biology: Challenging Life in Order to Grasp, Use, or Extend It," Biological Theory, 8(4), 2013 pp. 376-382.

[8] L. Bich and S. Green, "Is defining Life pointless? Operational definitions at the Frontiers of Biology," Synthese, 2017. doi:10.1007/s11229-017-1397-9.

[9] L. Martini and S. S. Mansy, "Cell-Like Systems with Riboswitch Controlled Gene Expression," Chemical Communications, 47, 2011 pp. 10734-10736.

[10] P. M. Gardner, K. Winzer and B. G. Davis, "Sugar Synthesis in a Protocellular Model Leads to a Cell Signalling Response in Bacteria.," Nature Chemistry, 1(5), 2009 pp. 377-383.

[11] G. Rampioni, F. Mavelli, L. Damiano, F. D’Angelo, M. Messina, L. Leoni and P. Stano, "A Synthetic Biology Approach to Bio-chem-ICT: First Moves Towards Chemical Communication between Synthetic and Natural Cells," Natural Computing, 13(3), 2014 pp. 333-349.

[12] R. Lentini, N. Martin, M. Forlin, L. Belmonte, J. Fontana, M. Cornella, L. Martini, S. Tamburini, W. E. Bentley, O. Jousson and S. Mansy, "Two-Way Chemical Communication between Artificial and Natural Cells," ACS Central Science, 3(2), 2017 pp. 117-123.

[13] M. Amos, P. Dittrich, J. McCaskill and S. Rasmussen, "Biological and Chemical Information Technologies," 
Procedia Computer Science, 7, 2011 pp. 56-60.

[14] P. Stano, Y. Kuruma and L. Damiano, "Synthetic Biology and (Embodied) Artificial Intelligence: Opportunities and Challenges," Adaptive Behavior, 26(1), 2018 pp. 41-44.

[15] P. Carrara, P. Stano and P. L. Luisi, "Giant Vesicles 'Colonies': A Model for Primitive Cell Communities," ChemBioChem, 13(10), 2012 pp. 1497-1502.

[16] Y. Qiao, M. Li, R. Booth and S. Mann, "Predatory Behaviour in Synthetic Protocell Communities," Nature Chemistry, 9(2), 2017 pp. 110-119.

[17] S. S. Mansy, "Non-living predators," Nature Chemistry, 9(2), 2017 pp. 107-108.

[18] T. Suda, M. Moore, T. Nakano, R. Egashira and A. Enomoto, "Exploratory Research on Molecular Communication between Nanomachines," in Proceedings of Genetic and Evolutionary Computation Conference (GECCO'05), Late Breaking Papers (F. Rothlauf, ed.), New York: ACM, 2005.

[19] L. Cronin, N. Krasnogor, B. G. Davis, C. Alexander, N. Robertson, J. H. G. Steinke, S. L. M. Schroeder, A. N. Khlobystov, G. Cooper, P. M. Gardner, P. Siepmann, B. J. Whitaker and D. Marsh, "The Imitation Game: A Computational Chemical Approach to Recognizing Life," Nature Biotechnology, 24(10), 2006 pp. 1203-1206.

[20] M. Forlin, R. Lentini and S. S. Mansy, "Cellular Imitations," Current Opinion in Chemical Biology, 16(5-6), 2012 pp. 586-592.

[21] F. J. Varela, Principles of Biological Autonomy, New York: North Holland, 1979.

[22] H. Maturana and F. J. Varela, Autopoiesis and Cognition: The Realization of the Living, Dordrecht: Reidel Publishing, 1980.

[23] S. A. Kauffman, Investigations. New York: Oxford University Press, 2000.

[24] A. Moreno and M. Mossio, Biological Autonomy: A Philosophical and Theoretical Enquiry, Dordrecht: Springer Netherlands, 2015.

[25] P. L. Luisi and F. J. Varela, "Self-Replicating Micelles-A Chemical Version of a Minimal Autopoietic System," Origins of Life and Evolution of the Biosphere, 19(6), 1989 pp. 633-643.

[26] P. L. Luisi, The Emergence of Life: From Chemical Origins to Synthetic Biology, Cambridge: Cambridge University Press, 2006.

[27] K. Ruiz-Mirazo and A. Moreno, "Basic Autonomy as a Fundamental Step in the Synthesis of Life," Artifificial Life, 10(3), 2004 pp. 235-259.

[28] J. Piaget, Biologie et Connaissance, Paris: Gallimard, 1967.

[29] M. Bitbol and P. L. Luisi, “Autopoiesis with or without Cognition: Defining Life at Its Edge," Journal of The Royal Society Interface, 1(1), 2004 pp. 99-107.

[30] L. Damiano and P. L. Luisi, "Towards an Autopoietic Redefinition of Life," Origins of Life and Evolution of the Biosphere, 40(2), 2010 pp. 145-9.

[31] L. Bich and L. Damiano, "Life, Autonomy and Cognition: An Organizational Approach to the Definition of the Universal Properties of Life," Origins of Life and Evolution of Biospheres, 42(5), 2012 pp. 389-397.

[32] L. Bich and A. Moreno, "The Role of Regulation in the Origin and Synthetic Modelling of Minimal Cognition," Biosystems, 148, 2016 pp. 12-21.

[33] J. Vallverdù, O. Castro, R. Mayne, M. Talanov, M. Levin, F. Baluska, Y. Gonji, A. Dussutour, H. Zenil and 
A. Adamatzky, "Slime Mould: The Fundamental Mechanisms of Biological Cognition," Biosystems, 165, 2018 pp. 57-70.

[34] L. Bich, M. Mossio, K. Ruiz-Mirazo and A. Moreno, "Biological Regulation: Controlling the System from Within," Biology and Philosophy, 31(2), 2016 pp. 237-265.

[35] H. Maturana and F. J. Varela, The Tree of Knowledge, Boston: Shambhala, 1987.

[36] R. Dawkins and J. R. Krebs, “Animal Signals: Information or Manipulation?,” in Behavioural Ecology: An Evolutionary Approach (R. Krebs and N. B. Davies, eds.) Sutherland, MA: Sinauer Associates, Inc., 1978 pp. 282-309.

[37] M. J. Owren, D. Rendall and M. J. Ryan, "Redefining Animal Signaling: Influence versus Information in Communication," Biology \& Philosophy, 25(5), 2010 pp. 755-780.

[38] E. Di Paolo, "On the Evolutionary and Behavioral Dynamics of Social Coordination: Models and Theoretical Aspects.," Ph.D. thesis, University of Sussex, UK, 1999.

[39] S. P. Diggle, A. Gardner, S. A. West and A. S. Griffin, "Evolutionary Theory of Bacterial Quorum Sensing: When Is a Signal Not a Signal?," Philosophical Transactions of the Royal Society B: Biological Sciences, 362(1483), 2007 pp. 1241-1249.

[40] T. C. Scott-Phillips, “Defining Biological Communication,” Journal of Evolutionary Biology, 21(2), 2008 pp. 387-395.

[41] M. Mossio, C. Saborido, and A. Moreno, "An Organizational Account of Biological Functions," The British Journal for the Philosophy of Science, 60(4), 2009 pp. 813-841.

[42] W. Christensen and M. Bickhard, "The Process Dynamics of Normative Function," The Monist, 85(1), 2002 pp. 3-28.

[43] G. Toepfer, "Teleology and Its Constitutive Role for Biology as the Science of Organized Systems in Nature," Studies in History and Philosophy of Biological and Biomedical Sciences, 43(1), 2012 pp. 113-119.

[44] M. Mossio and L. Bich, "What Makes Biological Organisation Teleological?", Synthese, 194(4), 2017 pp. 1089-1114. 